\title{
Unique cellular and humoral immunogenicity profiles generated by aerosol, intranasal, or parenteral vaccination in rhesus macaques
}

\author{
Diane L. Bolton, ${ }^{1+}$ Kaimei Song, ${ }^{1}$ Georgia D. Tomaras, ${ }^{2}$ Srinivas Rao, ${ }^{1 \S}$ Mario \\ Roederer ${ }^{1 \#}$
}




\section{Abstract}

3 Respiratory mucosa immunization is capable of eliciting both local and distal 4 mucosal immune responses; it is a potentially powerful yet largely unused modality for

5 vaccination against respiratory diseases. Targeting the lower versus upper airways by

6 aerosol delivery alters the immunogenicity profile of a vaccine, although the full extent of

7 this impact is not well characterized. We set out to define the cellular and humoral

8 response profiles elicited by immunization via intranasal, small aerosol droplets, and

9 large aerosol droplets. We compared responses following adenovirus-vectored

10 vaccination by these routes in macaques, either for the generation of primary immune

11 responses or for the boosting of previously primed systemic responses. Aerosol delivery

12 (4 or $10 \mu \mathrm{m}$ diameter droplets, addressing lower or upper airways, respectively) generated

13 the highest magnitude lung CD4 and CD8 T-cell responses, reaching 10-30\% vaccine-

14 specific levels in bronchoalveolar lavage cells. In contrast, intranasal delivery was less

15 immunogenic with $>10$-fold lower peak lung T-cell responses. Systemic (blood) T-cell

16 responses were only observed following $4 \mu \mathrm{m}$ aerosol (and parenteral) immunization,

17 while all delivery routes elicited similar humoral responses. These data demonstrate

18 distinct immune response profiles with each respiratory tract vaccination modality and

19 suggest that small droplet aerosol offers several immunological advantages over other

20 respiratory routes.

23 Keywords: Aerosol, nasal, vaccine delivery route, immunogenicity, rhesus macaque 


\section{Introduction}

4 sterilizing immunity, including influenza, respiratory syncytia virus, and tuberculosis.

5 Vaccines that elicit potent and durable immune responses in the respiratory mucosa have

6 the potential to reduce the global burden of these and other airborne diseases.

7 Immunization regimens delivered to the respiratory tract initiate immune responses in

8 mucosa-associated inductive sites and draining lymph nodes, resulting in robust local

9 cellular and humoral responses as well as distal genital responses via immune cell linkage

10 between these sites [1-3]. A better understanding of how airway mucosal delivery

11 modalities differ will inform multiple applications including vaccines, gene therapy, and

12 drug delivery.

13 Airway vaccination approaches explored to date include instillation in the nasal

14 cavity and aerosol delivery by nebulizer. These two approaches target different anatomic

15 sites, which may impact the magnitude and quality of immune responses as well as safety

16 profiles. The relative immunogenicity of different respiratory mucosal vaccine delivery

17 routes has not been thoroughly investigated. Studies in animal models and humans

18 suggest that upper and lower respiratory vaccinations elicit distinct immune profiles. For

19 example, systemic and mucosal antibody responses are greatly enhanced in mice when

20 intranasal viral-like particle (VLP) immunization is performed under anesthesia (akin to

21 aerosol delivery) rather than a conscious state $[\underline{4}, \underline{5}]$. This was attributed to greater

22 antigen deposition and uptake observed in the lungs of anesthetized animals. Similarly,

23 measles virus vaccine administered by nebulizer (aerosol) is more immunogenic and

24 achieves higher levels of protection against measles challenge in rhesus macaques than

25 vaccination targeting the upper respiratory tree $[\underline{6}, \underline{7]}$. Results from human clinical trials

26 with human papillomavirus VLP also indicate greater immunogenicity by lower airway

27 vaccination compared to nasal administration []ㅡ. Thus while several intranasal subunit

28 and live attenuated vaccines are very effective in humans [ㅇ-10], there is mounting

29 evidence that immunogenicity and efficacy may be further improved by aerosol delivery.

30 Additional investigation of how immune responses differ when elicited by different 
1 respiratory mucosal vaccination routes is warranted, including a better understanding of

2 the underlying immunogenicity mechanisms.

3 While intranasal immunization has been commonly studied in rodent models, the

4 caveat to the interpretation of these data is that much of the instilled vaccine enters the

5 lung, and thus induction occurs at both mucosa. The much larger physical separation of

6 the nasal cavity from the bronchi in primates and man likely impacts to a much greater

7 extent the immune profiles generated by separately addressing these two mucosal sites.

8 The objective of the present study was to directly compare immunogenicity of

9 several airway vaccination routes targeting different regions of the upper and lower

10 respiratory tract in rhesus macaques. We previously demonstrated that smaller aerosol

11 droplets (4 $\mu \mathrm{m}$ diameter) elicit greater systemic cellular and humoral responses than

12 larger, 10-11 $\mu \mathrm{m}$ droplets, despite similar responses in the respiratory tract [11]. We

13 directly compared the immunogenicity of vaccine delivery via the following four routes:

14 intranasal, $4 \mu \mathrm{m}$ aerosol ( $\mathrm{AE} 4 \mu \mathrm{m}$ ), $10 \mu \mathrm{m}$ aerosol (AE $10 \mu \mathrm{m}$ ), and parenteral. We tested

15 the ability of these delivery routes to elicit responses alone (i.e., priming) and following a

16 systemic DNA prime (i.e., heterologous boosting). We find that the latter greatly

17 enhances immunogenicity over mucosal delivery alone and is likely to be employed in

18 any mucosal vaccination approach [12-14]. Our studies reveal distinct profiles of mucosal

19 and systemic $\mathrm{T}$ cell responses across the vaccine delivery routes and highlight the

20 variability of immune induction within the respiratory mucosa. 
Animals and immunizations. Colony-bred Indian-origin female rhesus macaques

3 were immunized with three plasmid DNA priming immunizations four weeks apart,

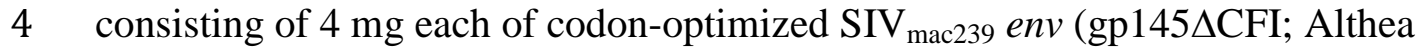

5 Technologies, CA). Immunogens were expressed within the vector pVR1012 under the

6 control of cytomegalovirus immediate-early enhancer, promoter, and first intron.

7 Delivery was intramuscular in the anterior quadriceps by Biojector. Recombinant

8 E1/E3/E4-deleted rAd5 constructs and virus stocks were generated as previously

9 described [15-17]. rAd5 expressing $\operatorname{GagPol}_{\text {SIV }}\left(1 \times 10^{10}\right)$ and $\operatorname{Env}_{\text {SIV }}\left(g p 140 ; 1 \times 10^{10}\right)$ was

10 administered eight weeks following the last DNA prime by one of four routes: intranasal

11 (IN), aerosol (AE) $4 \mu \mathrm{m}$ diameter droplets, AE $10 \mu \mathrm{m}$, or intramuscular (IM). IM was

12 performed by needle and syringe in the right quadriceps; AE by e-Flow ${ }^{\circledR}$ Nebulizer

13 System (PARI Pharma, Germany); and IN by instillation in the nasal cavity. Particle size

14 distributions generated by the e-Flow ${ }^{\circledR}$ were determined by the Aerodynamic Particle

15 Sizer® 3321 spectrometer (TSI, MN). For tracking studies, Fluoresbrite ${ }^{\circledR}$ Polychromatic

16 Red $0.5 \mu \mathrm{m}$ microspheres (Polysciences, Inc. Cat no. 19507-5) were delivered by AE at

$172.5 \times 10^{11}$ or $4 \times 10^{11}$ total particles. Animals were sacrificed 18 hours after bead delivery

18 for tissue collection and analysis of cellular bead uptake by flow cytometry. Single cell

19 suspensions were generated from respiratory tract tissue using a gentleMACS ${ }^{\mathrm{TM}}$

20 Dissociator.

21 Ethics Statement. All in vivo procedures were carried out in accordance to

22 institutional, local, state, and national guidelines and laws governing research in animals

23 including the Animal Welfare Act. Animal protocols and procedures were reviewed and

24 approved by the Animal Care and Use Committee (ACUC) of both the Vaccine Research

25 Center as well as the Institutional Animal Care and Use Committee of Bioqual, Inc.

26 where non-human primates were housed for the duration of the study. Bioqual Inc., and

27 the NIH are both accredited by the Association for Assessment and Accreditation of

28 Laboratory Animal Care (AAALAC) and are in full compliance with the Animal Welfare

29 Act and Public Health Service Policy on Humane Care and Use of Laboratory Animals.

30 Antibody measurements. SIV Gag-and Env-specific humoral IgG responses were

31 evaluated by a standardized binding antibody multiplex array as previously described [ $\underline{18}$, 
1 19]. Positive and negative monkey sera controls were used in each assay and the

2 midpoint titer $\left(\mathrm{EC}_{50}\right)$ of each sample was calculated using a 4 parameter logistic curve fit.

3 Positive responses to the vaccine were assessed as three-fold over pre-immune values and

4 at least 100 MFI. Rectal, nasal, and vaginal secretions were sampled by a modified wick

5 method using Weck-Cel Spears (Windsor Biomedical, Newton, $\mathrm{NH}$ ) as previously

6 described [20]. The antigen-specific IgA or IgG fluorescence intensity was divided by

7 the concentration of total $\operatorname{IgA}$ or IgG for each sample to obtain specific activity.

8 Adenoviral neutralizing titers were performed by the NIAID Vaccine Immune T-Cell and

9 Antibody Laboratory based on a published assay [21].

10 Cellular immune responses. BAL and peripheral blood were collected

11 longitudinally from animals following immunization. Single cell suspensions were

12 stimulated with overlapping peptide pools of SIV Env, Gag, or Pol at $2.0 \mu \mathrm{g} / \mathrm{ml}$ for 16

13 hours as previously described. Following stimulation, cells were labeled with cell surface

14 markers (CD4-Alexa700APC, CD8-QDot655, CD3-Cy7APC) and ViViD (to

15 discriminate live/dead cells). Intracellular cytokine staining was performed on fixed and

16 permeabilized (BD Cytofix/Cytoperm, Becton Dickenson) samples with IFN $\gamma$-FITC,

17 TNF $\alpha$-Cy7PE, and IL-2-PE. Samples were analyzed on an LSR II (Becton Dickenson)

18 and analyzed using FlowJo software (Tree Star, Inc.). Statistical analysis and display of

19 multicomponent distributions was performed with SPICE $\mathrm{v} 4$. 


\section{Results}

3 Localization of aerosol droplet uptake

$4 \quad$ We hypothesized that small aerosol (AE) droplets penetrate deep into the lower

5 respiratory tract while larger droplets are restricted to the head airways, based on

6 previous observations in humans and non-human primates [22-25]. Using the PARI e-

7 Flow nebulizer, we performed tracking studies to measure the deposition of differentially

8 sized aerosol droplets in rhesus macaques. Fluorescent polystyrene microspheres were

9 delivered by nebulizer-generated 4 or $10 \mu \mathrm{m}$ diameter droplets. Aerosol droplet sizes in

10 this range previously exhibited differential deposition properties and infection

11 transmission rates [22, 25]. Cells isolated from upper and lower respiratory tract tissue

12 were examined for bead uptake by flow cytometry $18 \mathrm{~h}$ after delivery. Cells positive for

13 microspheres were readily detected in BAL following AE delivery of both droplet sizes,

14 ranging from $0.4-26 \%$ of all cells (Figure 1A-B). Strikingly, the fluorescence profiles

15 revealed discrete cell populations containing anywhere from one to hundreds of

16 microspheres. Across the five animals studied, trends indicated greater microsphere

17 uptake following $4 \mu \mathrm{m} \mathrm{AE}$ in most tissues, particularly in BAL, trachea, and lung lobes

18 (Figure 1B). Cellular uptake of the aerosolized beads was further quantitated to account

19 for multiple microspheres per cell by calculating the total number of beads taken up by

20 all positive cells (Figure 1C). This analysis yielded similar results, confirming that $4 \mu \mathrm{m}$

21 AE delivers material more efficiently than $10 \mu \mathrm{m} \mathrm{AE}$ to cells residing throughout the

22 respiratory mucosa.

Mucosal (BAL) T cell responses

25 To compare humoral and cellular immune responses induced by AE droplets and

26 intranasal (IN) and intramuscular (IM) vaccine delivery routes, we immunized rhesus

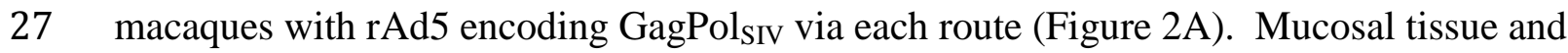

28 blood sampling was performed at regular intervals following immunization to measure

29 antigen-specific T-cells and antibodies across multiple tissues. GagPol-specific CD4 and

30 CD8 T-cells were quantified by ex vivo peptide pool stimulation followed by intracellular

31 cytokine staining and polychromatic flow cytometric detection (Figure 2). AE delivery 
1 elicited robust bronchoalveolar lavage (BAL) responses to GagPol, ranging from 10-30\%

2 of CD4 T-cells and up to 25\% of CD8 T-cells one month post-rAd5 (Figure 2B),

3 consistent with previous studies [11]. IN delivery also induced substantial BAL

4 responses, but these were significantly less than those elicited by $\mathrm{AE} 4 \mu \mathrm{m}$, ranging from

$5 \quad 0.5-5 \%$ for most animals. GagPol responses following IM delivery were generally

6 undetectable. This finding was inconsistent with 1-7\% responding BAL CD8 T-cells

7 observed previously following IM immunization $[11,12]$, and may be due to original

8 antigenic sin interference from rAd5-Env co-administered with rAd5-GagPol (see

9 below). Interpretation of the unprimed IM regimen in this experiment may thus be

10 confounded. CD8 T-cell responses in a subset of animals from the $10 \mu \mathrm{m} \mathrm{AE}$ and IN

11 groups were also unexpectedly low. Taken together, along with our prior studies in

12 which IN and IM delivery exhibit similar BAL T-cell priming with slightly greater

13 responses elicited by IN $[\underline{11}, \underline{12}]$, we observed the following hierarchy for priming lung

14 mucosal $\mathrm{T}$ cell responses: $\mathrm{AE} 4 \mu \mathrm{m} \cong \mathrm{AE} 10 \mu \mathrm{m}>\mathrm{IN} \cong \mathrm{IM}$. Following response peak,

15 GagPol-specific T-cells contracted to similarly low levels ranging from $0-1.5 \%$ for all

16 groups (week 29 and 39, data not shown).

17 To test the boosting capability of each route, responses to a different immunogen,

18 Env $_{\text {SIV }}$, were first primed with DNA intramuscularly three times at monthly intervals

19 prior to boosting with rAd5 encoding Env via the various routes (Figure 2A). CD4 and

20 CD8 responses to the DNA prime were modest (Figure 2C), as expected. Mucosal T-cell

21 responses elicited by the rAd5 boost were similar in magnitude to unprimed responses by

22 the same route (Figure $2 \mathrm{~B}$ and $[\underline{11}, \underline{12}]$ ). AE rAd5 boosting elicited the largest BAL

23 response, again ranging from 10-30\% of both CD4 and CD8 T-cell subsets. By contrast,

24 responses to IN and IM delivery comprised 0-2\% of CD4 T-cells and 0-5\% of CD8 T-

25 cells. Peak responses were typically detected four weeks post-rAd5 for the AE and IM

26 groups, while IN responses were often delayed, consistent with previous experiments

27 (data not shown). The boosting capabilities can thus be summarized as follows: AE 10 $\mu \mathrm{m}$

$28 \cong \mathrm{AE} 4 \mu \mathrm{m}>\mathrm{IN} \cong \mathrm{IM}$.

29 For comparison, peak primed and unprimed mucosal BAL responses were plotted

30 together in Figure 2D. CD4 T-cell responses were similar in magnitude for Env and

31 GagPol for all immunization routes, suggesting that systemic DNA did not prime CD4 
1 BAL T-cells. The effect of DNA priming on CD8 T-cell responses was less clear due to

2 the atypical unprimed GagPol response observed for most delivery conditions.

3 Remarkably, CD8 responses to AE $4 \mu$ m were robust for both Env and GagPol.

4 Combined, AE rAd5 was more immunogenic than IN delivery in the respiratory mucosa,

5 regardless of priming status.

Peripheral blood T-cell responses

8 To study elicitation of systemic antigen-specific T-cells, we performed similar

9 ICS analyses on PBMC samples. Low-magnitude, transient CD4 T-cell responses were

10 observed to the unprimed Gag antigen for IM and both AE routes (Figure 3A), while only

11 IM vaccination generated significant unprimed CD8 T-cell responses. These responses

12 were generally below the limit of detection one month after rAd5. PBMC responses

13 elicited by $\mathrm{AE} 4 \mu \mathrm{m}$ and $10 \mu \mathrm{m}$ immunization were not significantly different, whereas we

14 previously observed higher magnitude responses by $\mathrm{AE} 4 \mu \mathrm{m}$ [11].

15 In the setting of systemic DNA-priming, all routes showed boosting CD4

16 responses relative to pre-Ad levels (IM, p=0.04; $\mathrm{AE} 10 \mu \mathrm{m}, \mathrm{p}=0.15$; $\mathrm{AE} 4 \mu \mathrm{m}, \mathrm{p}=0.08$; $\mathrm{IN}$,

$17 \mathrm{p}=0.33$, wk 16 vs. 18 ; Figure $3 \mathrm{~B}$ ). By contrast, there was a striking difference among the

18 delivery routes with respect to CD8 T-cell boosting. Only IM and AE $4 \mu \mathrm{m}$ boosted

19 DNA-primed responses (IM, p=0.04, AE, p=0.09, wk 16 vs. 18), achieving 0.2-2\% Env-

20 specific CD8 T-cells (Figure 3B-C). Blood CD8 T-cell responses were not enhanced by

21 either $\mathrm{AE} 10 \mu \mathrm{m}$ or IN rAd5 delivery. Thus $\mathrm{AE} 4 \mu \mathrm{m}$ is more similar to IM immunization

22 with respect to boosting systemic CD8 T-cell responses, while AE $10 \mu \mathrm{m}$ and IN were

23 less immunogenic.

Quality of cellular responses

26 Since T-cell response quality correlates with disease protection in multiple animal

27 models and human infections [26-28], we analyzed the combination of cytokines

28 expressed by antigen-specific T-cells in each vaccine group. Three months after rAd5

29 primary immunization, the quality of the BAL memory CD4 T-cell (GagPol-specific)

30 response differed between the $\mathrm{AE}$ groups and the $\mathrm{IM}$ group $(\mathrm{p}=0.05, \mathrm{AE} 4 \mu \mathrm{m} ; \mathrm{p}=0.06$,

$31 \mathrm{AE} 10 \mu \mathrm{m}$, Figure S1A). AE delivery shifted responses towards greater polyfunctionality 
1 as measured by simultaneous co-expression of IFN $\gamma$, IL-2, and TNF by a larger fraction

2 of the antigen-specific cells. No significant differences were observed between vaccine

3 delivery routes for $\mathrm{CD} 8 \mathrm{~T}$ cells or at other time points.

$4 \quad$ We also assessed whether DNA priming influenced the quality of the response for

5 each vaccine delivery route. At peak, BAL CD8 T-cell responses to IN immunization

6 were more polyfunctional with DNA priming than without the systemic prime (Figure

7 S1B). BAL responses were skewed towards IL-2 single positive and IL2+TNF $\alpha+$ cells

8 in the absence of DNA priming, while the primed response was comprised largely of

9 IL2+TNF $\alpha+\mathrm{INF} \gamma+$ and IFN $\gamma$ single-positive cells. This is consistent with DNA priming

10 driving the $\mathrm{T}$ cell response towards a more differentiated phenotype [29]. Response

11 quality for other vaccination routes was not influenced by the systemic prime.

\section{Humoral responses}

14 Env- and Gag-specific antibodies were measured in plasma and mucosal

15 secretions following the DNA prime and rAd5 immunizations. DNA alone elicited

16 significant plasma gp120-specific $\operatorname{IgG}$ and $\operatorname{IgA}$ responses ( $\mathrm{p}<0.01$ and $\mathrm{p}<0.03$,

17 respectively, Figure 4A), while nasal, vaginal, and rectal responses were low or

18 undetectable (Figure 4B). Boosting with the viral vector significantly increased Env-

19 specific plasma IgG or IgA for all delivery routes ( $\mathrm{p}<0.05)$. Nasal, vaginal, and rectal

20 mucosal responses were also boosted by rAd5 relative to post-DNA values, although

21 statistical significance was not achieved for some delivery routes due to within group

22 variability (Figure 4B). Similar trends were observed for antibodies specific for gp130

23 and gp140 Env antigens (data not shown). gp120-specific IgG or IgA mucosal responses

24 generally were not significantly different between vaccination groups following rAd5

25 immunization, with the exception of greater nasal $\mathrm{IgG}$ following IM versus $\mathrm{AE} 10 \mu \mathrm{m}$

26 delivery and greater vaginal IgA following IM versus nasal delivery. Surprisingly, no

27 significant antibody responses to the Gag immunogen were detected, likely due in part to

28 greater inherent Env humoral immunogenicity.

29 Circulating Env-specific humoral responses elicited by the rAd5 boost were

30 further quantitated by titrating plasma to determine the half maximal effective

31 concentration $\left(\mathrm{EC}_{50}\right)$ for responses to three versions of the Env antigen. IM delivery 
1 typically induced the largest plasma IgG responses, with greater gp 140 -specific $\operatorname{IgG}$ than

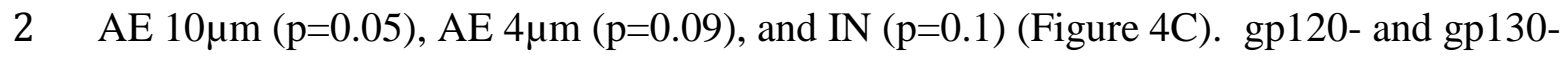

3 specific responses followed a similar pattern. Thus IM delivery potentially offers a slight

4 advantage with respect to boosting plasma IgG magnitude, but overall responses were

5 similar among the groups.

6 We previously reported that little or no humoral response is generated to the viral

7 vector when it is administered $\mathrm{AE}$ [11]. To compare the anti-vector response across the

8 delivery routes used here, we measured serum Ad5 neutralizing antibodies four weeks

9 after rAd5 vaccination. As expected, Ad5 titers were robust following IM delivery: 90\%

10 inhibitory plasma concentrations $\left(\mathrm{IC}_{90}\right)$ values ranged from $1000-4000^{-1}$ in all animals

11 (Figure 4D). IN delivery was less potent, but low-level responses were mounted in three

12 of four animals. By contrast, AE was largely silent with respect to Ad5 immunogenicity

13 with undetectable titers in seven of eight animals. Taken together with the humoral

14 responses elicited against the Env insert, these data indicate that mucosal airway and

15 parenteral vaccination achieve similar humoral responses to the immunogen, but the latter

16 is much more immunogenic with respect to anti-vector responses. Between the mucosal

17 routes, IN is more likely to generate vector-specific antibodies than AE. 


\section{Discussion}

Respiratory mucosal vaccination has been well tolerated, immunogenic, and

4 efficacious in humans as well as animal models of disease. The immunologic advantage

5 gained by targeting vaccines to different regions of the respiratory tract, however, is not

6 clear. Here we report distinct immune profiles following AE $4 \mu \mathrm{m}, \mathrm{AE} 10 \mu \mathrm{m}$, and IN

7 delivery of rAd5 vaccine vector in rhesus macaques (summarized in Table 1). $\mathrm{AE}$

8 vaccination with either 4 or $10 \mu \mathrm{m}$ diameter droplets elicits 1-2 log greater peak lung

$9 \mathrm{CD} 4$ and CD8 T-cell responses than IN, which exhibited immunogenicity more similar to

10 IM than AE. This was observed for both unprimed and systemic DNA-primed responses.

11 BAL CD4 T-cell responses were generally not primed by DNA, regardless of vaccination

12 route. A different immunogenicity hierarchy was observed for blood T-cell responses:

$13 \mathrm{IM}$ and $\mathrm{AE} 4 \mu \mathrm{m}$ robustly boosted CD8 responses, while little boosting was observed by

$14 \mathrm{AE} 10 \mu \mathrm{m}$ and IN. Tracking of AE droplets within respiratory mucosa further

15 distinguished $\mathrm{AE} 4 \mu \mathrm{m}$ and $10 \mu \mathrm{m}$ immunization, with greater cellular uptake of material

16 delivered by $4 \mu \mathrm{m}$ droplets. Mucosal and plasma humoral responses to the Env

17 immunogen were similar regardless of the rAd5 delivery route. Upon longer follow up,

18 systemically primed AE generates more durable rectal IgA titers than IM vaccination

19 with the same prime [12]. Taken together, these data, combined with our previous T-cell

20 priming observations $[\underline{11}, \underline{12}]$, suggest that while both $4 \mu \mathrm{m}$ and $10 \mu \mathrm{m} \mathrm{AE}$ are far more

21 immunogenic than IN, only $4 \mu \mathrm{m}$ AE elicits robust peripheral T-cells.

22 Our tracking studies revealed greater cellular uptake of material delivered by AE

$234 \mu \mathrm{m}$ than $\mathrm{AE} 10 \mu \mathrm{m}$, particularly among cells residing in the lower respiratory tract. Our

24 data indicate that more antigen-presenting cells (APC) within the respiratory mucosa are

25 exposed to and engulf antigen when exposed to smaller aerosols. In turn, this likely

26 results in a higher frequency of APC in draining lymph nodes and a larger quantity of

27 antigen presented by each APC. Deeper penetration of the $4 \mu \mathrm{m}$ aerosol droplets may

28 also target APC that drain to more distal or distinct lymph nodes that alter the trafficking

29 of responding antigen-specific lymphocytes.

30 T-cell and antibody responses to the unprimed GagPol rAd5 insert were limited

31 and lower than our previous observations reporting robust cellular and humoral responses 
1 elicited by the same rAd5-GagPol vaccination in both unprimed and DNA-primed

2 settings $[11,12]$. GagPol-specific responses were thus impaired in this study. Inhibition

3 was possibly due to "original antigenic sin" from the prior exposure to Env DNA: i.e., the

4 priming immunization resulted in Env-dominated responses when both GagPol and Env

5 were co-delivered as a boost. Similar results have been previously noted for cellular

6 responses [30-33].

$7 \quad$ rAd5 vector-specific antibodies were consistently elicited by IM and IN, but not

8 AE. While vector-specific antibodies are not typically elicited by AE immunization [11],

9 one animal immunized by AE $4 \mu \mathrm{m}$ in this study mounted Ad5-specific titers. This

10 animal was among the strongest responders to the vaccination regimen within the $\mathrm{AE}$

$114 \mu \mathrm{m}$ group, with the largest CD4 $\mathrm{T}$ cell response to the DNA prime in both BAL and

12 PBMC. These data suggest that robust CD4 helper $\mathrm{T}$ cells generated by the systemic

13 prime specific for the insert encoded by a subsequent viral vector boost may facilitate

14 development of vector-specific antibody responses upon AE boosting.

15 In summary, we show that small droplet aerosols generate robust mucosal $\mathrm{T}$ cell

16 responses and, when primed systemically, boost both peripheral $\mathrm{T}$ cells and humoral

17 responses. Similar magnitude lung $\mathrm{T}$ cell responses are generated by small droplet

18 aerosols of rAd35 [11, 34] or Bacillus Calmette-Guérin (M. Roederer, unpublished),

19 suggesting that these findings may extend to other vectors and vaccine platforms. Given

20 good safety profiles of aerosol delivery in both humans and non-human primates, the

21 historical success of aerosol vaccination with live-attenuated measles virus in children [ $\underline{3}$,

$22 \underline{35}, \underline{36}$, and the promising results presented here, further consideration of aerosol

23 immunization is warranted for elicitation of durable and robust mucosal immunity.

24 These findings are pertinent for strategies to achieve mucosal immunity using

25 heterologous systemic/mucosal vaccination. 


\section{Figure Legends}

3 Figure 1. Differential uptake of aerosolized micro-beads with 4 and $10 \mu \mathrm{m}$ aerosol

4 droplets. Macaques received fluorescent particles by either 4 or $10 \mu \mathrm{m}$ nebulizers and

5 were sacrificed $18 \mathrm{~h}$ later for bead uptake in respiratory tract tissues. (A) Flow cytometric

6 analysis of ungated single cells isolated from the indicated tissue is shown for two

7 representative animals; $4 \mu \mathrm{m}$ (middle), $10 \mu \mathrm{m}$ (bottom), and negative control BAL from an

8 unexposed animal (top). Cellular uptake of red fluorescent microspheres is depicted by

9 the gated population, with percent positive indicated. Fluorescence at $450 \mathrm{~nm}$ is plotted

10 as a negative control, although some spill over or increased autofluorescence is observed

11 at this wavelength. Gray arrow indicates cell population containing a single microsphere.

12 (B) The percentage of cells positive for fluorescent microspheres by flow cytometric

13 analysis is plotted for five animals $(n=3, \operatorname{AE} 4 \mu \mathrm{m} ; \mathrm{n}=2, \mathrm{AE} 10 \mu \mathrm{m})$ across the indicated

14 tissues. Symbols represent individual animals. (C) Total microsphere uptake is plotted

15 as in (B). Uptake was calculated as follows: (fraction cells containing $\geq 1$ microsphere $\mathrm{x}$

16 mean G560 fluorescence of all positive cells) / (mean G560 fluorescence of cells

17 containing one microsphere).

19 Figure 2. Airway mucosal T-cell response magnitude. (A) Experimental vaccination

20 scheme. Sixteen rhesus macaques were immunized with three DNA primes encoding

21 Env at one-month intervals. Eight weeks after the last DNA prime, a mixture of two

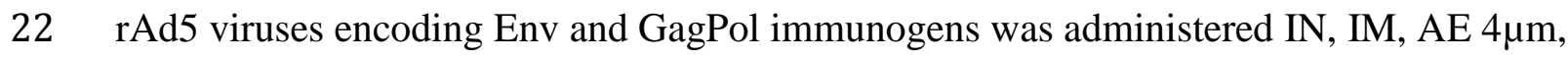

23 or $\mathrm{AE} 10 \mu \mathrm{m}(\mathrm{n}=4$ per group). Antigen-specific T-cells were measured throughout the

24 vaccination regimen in BAL by in vitro peptide stimulation and intracellular Th1

25 cytokine staining and flow cytometric quantitation. Unprimed GagPol-specific (B) and

26 DNA-primed Env-specific (C) $\mathrm{CD}^{+}$(top) and $\mathrm{CD}^{+}$(bottom) T-cell responses are shown

27 for each vaccination group at the indicated time point. Statistically significant $(\mathrm{p}<0.05$,

28 Wilcoxon rank-sum test) responses relative to the pre-immune measurement for each

29 vaccine group are indicated. (D) Direct comparison of DNA-primed and unprimed BAL

30 responses four weeks after rAd5 vaccination (week 20) is shown. 
1 Figure 3. Systemic T-cell response magnitude. Antigen-specific T-cell responses in

2 PBMC were measured and plotted as in Figure 2. The unprimed GagPol-specific (A) and

3 DNA-primed Env-specific (B) PBMC responses are shown. Statistically significant

$4 \quad(p<0.05$, Wilcoxon rank-sum test) responses relative to the pre-immune measurement for

5 each vaccine group are indicated. (C) DNA-primed and unprimed PBMC responses are

6 compared directly at peak (two weeks after rAd5, or week 18).

8 Figure 4. Systemic and mucosal humoral responses. (A-B) Env- and Gag-specific

9 antibodies were measured in plasma and mucosal (nasal, vaginal, rectal) secretions by

10 Luminex. (A) gp120-specific plasma IgG (top) and IgA (bottom) are plotted for each

11 animal as mean fluorescence intensity (MFI). Statistically significant $(\mathrm{p}<0.05)$ responses

12 relative to the post-DNA measurement for each vaccine group are indicated along the top

13 (Wilcoxon rank-sum test). "Post-DNA" and "post-Ad" refer to study week 14 (6 weeks

14 post-DNA) and 20 (4 weeks post Ad), respectively. Gray bars reflect the interquartile

15 range for each group. (B) Nasal, vaginal, and rectal $\operatorname{IgG}$ (top) and $\operatorname{IgA}$ (bottom) are

16 plotted as specific activity (binding units / total $\mathrm{Ig}(\mu \mathrm{g} / \mathrm{ml})$, where binding units =

17 MFI*dilution). Between group comparisons within a time point are indicated by lines

18 spanning group with significantly different responses. (C) Week 20 plasma IgG $\mathrm{EC}_{50}$

19 values for the indicated Env antigens were determined using a 4 parameter logistic curve

20 fit model. (D) The plasma 90\% adenovirus neutralizing titer was determined for each

21 animal before and 4 weeks after the rAd5 immunization and plotted as the reciprocal

22 dilution.

24 Supplemental Figure S1. T-cell response quality. The combination of cytokines

25 expressed by antigen-specific cells in response to cognate peptide stimulation was

26 measured by flow cytometry as in Figure 2. The proportion of the total antigen-specific

27 T-cell population expressing each possible combination of cytokines is shown as a pie

28 chart for each vaccination group; colored wedges correspond to cytokine profile indicated

29 at right. (A) Unprimed BAL CD4 ${ }^{+}$T-cell quality at memory (13 weeks post-rAd5) for

30 the Gag/Pol-specific response is shown. (B) BAL $\mathrm{CD}^{+}{ }^{+}$-cell quality at peak (4 weeks 
1 post-rAd5) for the unprimed (Gag) and DNA primed (Env) responses for each rAd5

2 vaccination route. Significantly different pie charts are indicated with p-values. 
1 Table 1. Summary of immune response magnitude by vaccine delivery route

\begin{tabular}{|c|c|c|c|c|c|c|c|c|c|}
\hline \multirow[b]{3}{*}{ Immunization } & \multicolumn{4}{|c|}{ Systemic* } & \multirow{2}{*}{\multicolumn{2}{|c|}{$\frac{\mathrm{BAL}}{\mathrm{T} \text { cell }}$}} & \multirow{2}{*}{\multicolumn{2}{|c|}{$\frac{\text { Mucosal }}{\text { Antibody }}$}} & \multirow{3}{*}{$\begin{array}{l}\text { Vector- } \\
\text { specific } \\
\text { neut. Ab }\end{array}$} \\
\hline & \multicolumn{2}{|c|}{$\mathrm{T}$ cell } & \multicolumn{2}{|c|}{ Antibody } & & & & & \\
\hline & Primary & Boost & Primary** & Boost & Primary** & Boost & Primary & Boost & \\
\hline DNA (IM) & - & n.a. & ++ & n.a. & + & n.a. & - & n.a. & n.a. \\
\hline Ad IM & +++ & ++ & ++ & +++ & ++ & ++ & n.a. & ++ & +++ \\
\hline Ad IN & + & + & n.a. & ++ & ++ & ++ & n.a. & + & ++ \\
\hline Ad AE $4 \mu \mathrm{m}$ & ++ & ++ & ++ & ++ & ++++ & ++++ & n.a. & $+{ }^{* * * *}$ & + \\
\hline Ad AE $10 \mu \mathrm{m}$ & ++ & + & + & ++ & +++ & ++++ & n.a. & + & - \\
\hline
\end{tabular}

2 *Immune responses were ranked by magnitude across all animals, followed by averaging the rank within 3 each vaccine group.

$4 \quad * * \mathrm{IM}$ and $\mathrm{AE}$ rankings include historic data $[11,12]$ due to atypical Gag responses in this study.

$5 \quad * * *$ Distal mucoasl IgA are more durable following rAd5 boost via AE 4um than IM [12].

6 n.a. $=$ not assessed

7 


\section{Acknowledgments}

2

3 We are grateful to Dr. Bob Bailer of the Vaccine Research Center (VRC) Immunology

4 Core Laboratory for adenovirus serology; JP Todd of the VRC Laboratory of Animal

5 Medicine for animal protocol preparation, animal procedure scheduling, and

6 administering immunizations; the VRC Flow Cytometry Core for maintenance of LSR II

7 flow cytometric instrumentation; members of the VRC ImmunoTechnology Section for

8 helpful scientific discussions; Judith T. Lucas, Yong Lin, Vicki C. Ashley, and R. Glenn

9 Overman for expert technical assistance; and BIOQUAL, Inc. for animal housing,

10 handling, and tissue collection.

11

12 This work was supported by the Intramural Research Program of the Vaccine Research

13 Center, NIAID, NIH and by a cooperative agreement (W81XWH-07-2-

14 0067) between the Henry M. Jackson Foundation for the Advancement of Military

15 Medicine, Inc., and the U.S. Department of Defense (DOD). The views expressed are

16 those of the authors and should not be construed to represent the positions of the US

17 Army or the Department of Defense.

18

19

20

21 
[1] Holmgren J, Czerkinsky C. Mucosal immunity and vaccines. Nature medicine. 2005;11:S45-53.

5 [2] Johansson EL, Wassen L, Holmgren J, Jertborn M, Rudin A. Nasal and vaginal vaccinations have differential effects on antibody responses in vaginal and cervical secretions in humans. Infection and immunity. 2001;69:7481-6. [3] Nardelli-Haefliger D, Lurati F, Wirthner D, Spertini F, Schiller JT, Lowy DR, et al. Immune responses induced by lower airway mucosal immunisation with a human papillomavirus type 16 virus-like particle vaccine. Vaccine. 2005;23:3634-41. [4] Balmelli C, Roden R, Potts A, Schiller J, De Grandi P, Nardelli-Haefliger D. Nasal immunization of mice with human papillomavirus type 16 virus-like particles elicits neutralizing antibodies in mucosal secretions. Journal of virology. 1998;72:8220-9. [5] Revaz S, Dudler J. [Clinical manifestations of gout]. Revue medicale suisse. 2007;3:728-30.

[6] de Swart RL, Kuiken T, Fernandez-de Castro J, Papania MJ, Bennett JV, Valdespino JL, et al. Aerosol measles vaccination in macaques: preclinical studies of immune responses and safety. Vaccine. 2006;24:6424-36. [7] de Swart RL, LiCalsi C, Quirk AV, van Amerongen G, Nodelman V, Alcock R, et al. Measles vaccination of macaques by dry powder inhalation. Vaccine. 2007;25:118390.

[8] Bergquist C, Lagergard T, Holmgren J. Anticarrier immunity suppresses the antibody response to polysaccharide antigens after intranasal immunization with the polysaccharide-protein conjugate. Infection and immunity. 1997;65:1579-83. [9] Mills KH, Cosgrove C, McNeela EA, Sexton A, Giemza R, Jabbal-Gill I, et al. Protective levels of diphtheria-neutralizing antibody induced in healthy volunteers by unilateral priming-boosting intranasal immunization associated with restricted ipsilateral mucosal secretory immunoglobulin a. Infection and immunity. 2003;71:726-32.

[10] Belshe R, Lee MS, Walker RE, Stoddard J, Mendelman PM. Safety, immunogenicity and efficacy of intranasal, live attenuated influenza vaccine. Expert review of vaccines. 2004;3:643-54.

[11] Song K, Bolton DL, Wei CJ, Wilson RL, Camp JV, Bao S, et al. Genetic immunization in the lung induces potent local and systemic immune responses. Proceedings of the National Academy of Sciences of the United States of America. 2010;107:22213-8.

[12] Bolton DL, Song K, Wilson RL, Kozlowski PA, Tomaras GD, Keele BF, et al. Comparison of systemic and mucosal vaccination: impact on intravenous and rectal SIV challenge. Mucosal immunology. 2012;5:41-52.

[13] Wei CJ, Boyington JC, McTamney PM, Kong WP, Pearce MB, Xu L, et al. Induction of broadly neutralizing H1N1 influenza antibodies by vaccination. Science. 2010;329:1060-4.

44 responses after intravaginal immunisation with trimeric HIV-1 CN54 clade C gp140 
1 in Carbopol gel are augmented by systemic priming or boosting with an adjuvanted

2 formulation. Vaccine. 2011;29:1421-30.

3 [15] Brough DE, Lizonova A, Hsu C, Kulesa VA, Kovesdi I. A gene transfer vector-cell

4 line system for complete functional complementation of adenovirus early regions E1

5 and E4. Journal of virology. 1996;70:6497-501.

6 [16] Gall JG, Lizonova A, EttyReddy D, McVey D, Zuber M, Kovesdi I, et al. Rescue and

7 production of vaccine and therapeutic adenovirus vectors expressing inhibitory

8 transgenes. Molecular biotechnology. 2007;35:263-73.

9 [17] Rasmussen H, Rasmussen C, Lempicki M, Durham R, Brough D, King CR, et al.

10 TNFerade Biologic: preclinical toxicology of a novel adenovector with a radiation-

11 inducible promoter, carrying the human tumor necrosis factor alpha gene. Cancer

12 gene therapy. 2002;9:951-7.

13 [18] Tomaras GD, Yates NL, Liu P, Qin L, Fouda GG, Chavez LL, et al. Initial B-cell

14 responses to transmitted human immunodeficiency virus type 1: virion-binding

15 immunoglobulin M (IgM) and IgG antibodies followed by plasma anti-gp41

16 antibodies with ineffective control of initial viremia. Journal of virology.

17 2008;82:12449-63.

18 [19] Yates NL, Stacey AR, Nolen TL, Vandergrift NA, Moody MA, Montefiori DC, et al.

19 HIV-1 gp41 envelope IgA is frequently elicited after transmission but has an initial secretions and analysis of mucosal HIV antibody. J Acquir Immune Defic Syndr. 2000;24:297-309.

[21] Sprangers MC, Lakhai W, Koudstaal W, Verhoeven M, Koel BF, Vogels R, et al. Quantifying adenovirus-neutralizing antibodies by luciferase transgene detection: addressing preexisting immunity to vaccine and gene therapy vectors. J Clin Microbiol. 2003;41:5046-52.

[22] Day WC, Berendt RF. Experimental tularemia in Macaca mulatta: relationship of aerosol particle size to the infectivity of airborne Pasteurella tularensis. Infection and immunity. 1972;5:77-82.

[23] Druett HA, Henderson DW, Packman L, Peacock S. Studies on respiratory infection. I. The influence of particle size on respiratory infection with anthrax spores. The Journal of hygiene. 1953;51:359-71.

[24] ICRP. Human respiratory tract model for radiological protection.: Elsevier Health Sciences; 1994.

[25] Cheng YS, Irshad H, Kuehl P, Holmes TD, Sherwood R, Hobbs CH. Lung deposition of droplet aerosols in monkeys. Inhalation toxicology. 2008;20:1029-36. [26] Darrah PA, Patel DT, De Luca PM, Lindsay RW, Davey DF, Flynn BJ, et al. Multifunctional TH1 cells define a correlate of vaccine-mediated protection against Leishmania major. Nat Med. 2007;13:843-50.

[27] Harari A, Petitpierre S, Vallelian F, Pantaleo G. Skewed representation of functionally distinct populations of virus-specific CD4 T cells in HIV-1-infected subjects with progressive disease: changes after antiretroviral therapy. Blood.

$45 \quad 2004 ; 103: 966-72$. 
1 [28] Lin L, Finak G, Ushey K, Seshadri C, Hawn TR, Frahm N, et al. COMPASS

2 identifies T-cell subsets correlated with clinical outcomes. Nat Biotechnol.

3 2015;33:610-6.

4 [29] Seder RA, Darrah PA, Roederer M. T-cell quality in memory and protection:

5 implications for vaccine design. Nature reviews Immunology. 2008;8:247-58.

6 [30] Da Silva DM, Pastrana DV, Schiller JT, Kast WM. Effect of preexisting

7 neutralizing antibodies on the anti-tumor immune response induced by chimeric

8 human papillomavirus virus-like particle vaccines. Virology. 2001;290:350-60.

9 [31] Liu XS, Dyer J, Leggatt GR, Fernando GJ, Zhong J, Thomas R, et al. Overcoming

10 original antigenic sin to generate new CD8 T cell IFN-gamma responses in an

11 antigen-experienced host. Journal of immunology. 2006;177:2873-9.

12 [32] Zompi S, Harris E. Original antigenic sin in dengue revisited. Proc Natl Acad Sci

13 U S A. 2013;110:8761-2.

14 [33] Good MF, Zevering Y, Currier J, Bilsborough J. 'Original antigenic sin', T cell

15 memory, and malaria sporozoite immunity: an hypothesis for immune evasion.

16 Parasite Immunol. 1993;15:187-93.

17 [34] Darrah PA, Bolton DL, Lackner AA, Kaushal D, Aye PP, Mehra S, et al. Aerosol

18 vaccination with AERAS-402 elicits robust cellular immune responses in the lungs of

19 rhesus macaques but fails to protect against high-dose Mycobacterium tuberculosis

20 challenge. J Immunol. 2014;193:1799-811.

21 [35] Dilraj A, Cutts FT, de Castro JF, Wheeler JG, Brown D, Roth C, et al. Response to

22 different measles vaccine strains given by aerosol and subcutaneous routes to

23 schoolchildren: a randomised trial. Lancet. 2000;355:798-803.

24 [36] Hokey DA, Wachholder R, Darrah PA, Bolton DL, Barouch DH, Hill K, et al. A

25 nonhuman primate toxicology and immunogenicity study evaluating aerosol

26 delivery of AERAS-402/Ad35 vaccine: Evidence for transient $t$ cell responses in

27 peripheral blood and robust sustained responses in the lungs. Hum Vaccin

28 Immunother. 2014;10:2199-210.

29 


\section{Figure 1}

A

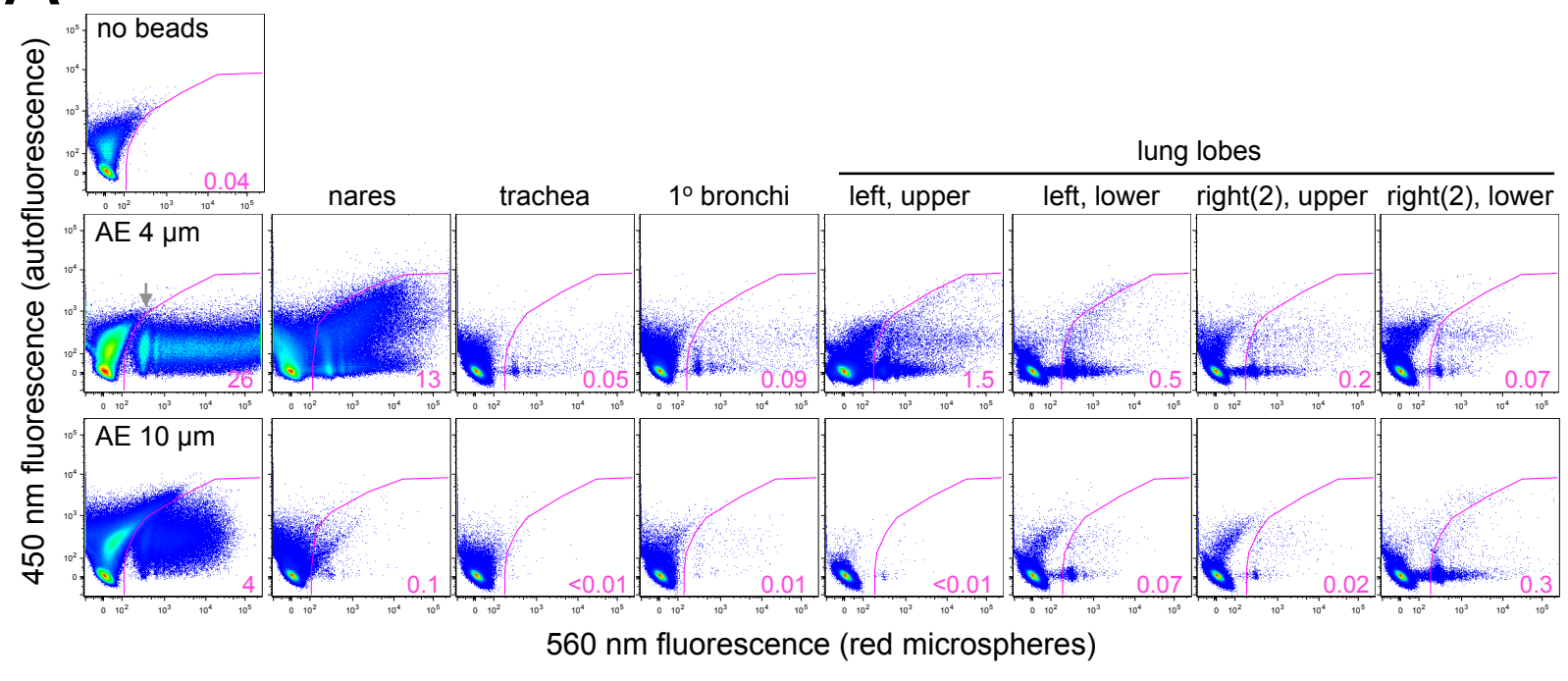

B

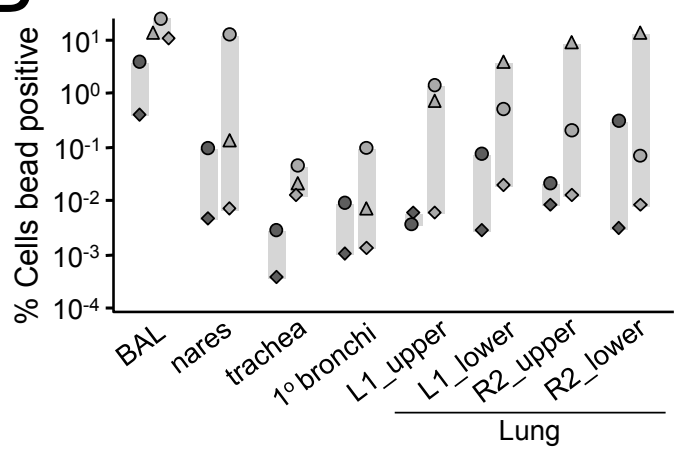

C

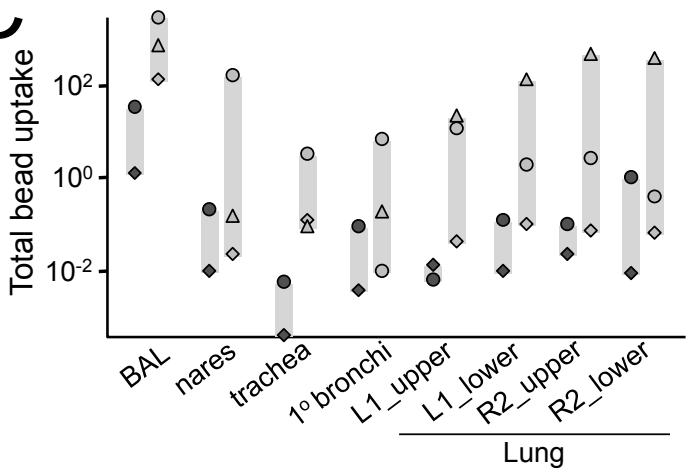

$\square \mathrm{AE} 10 \mu \mathrm{m}$

$\square \mathrm{AE} 4 \mu \mathrm{m}$ 


\section{Figure 2}

A

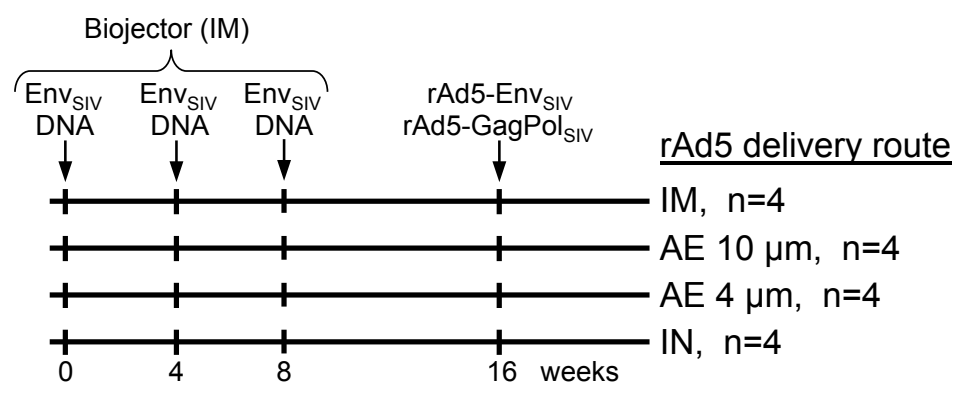

\begin{tabular}{c|l|l|l} 
Env & & Expected immune \\
& & & \\
responses
\end{tabular}

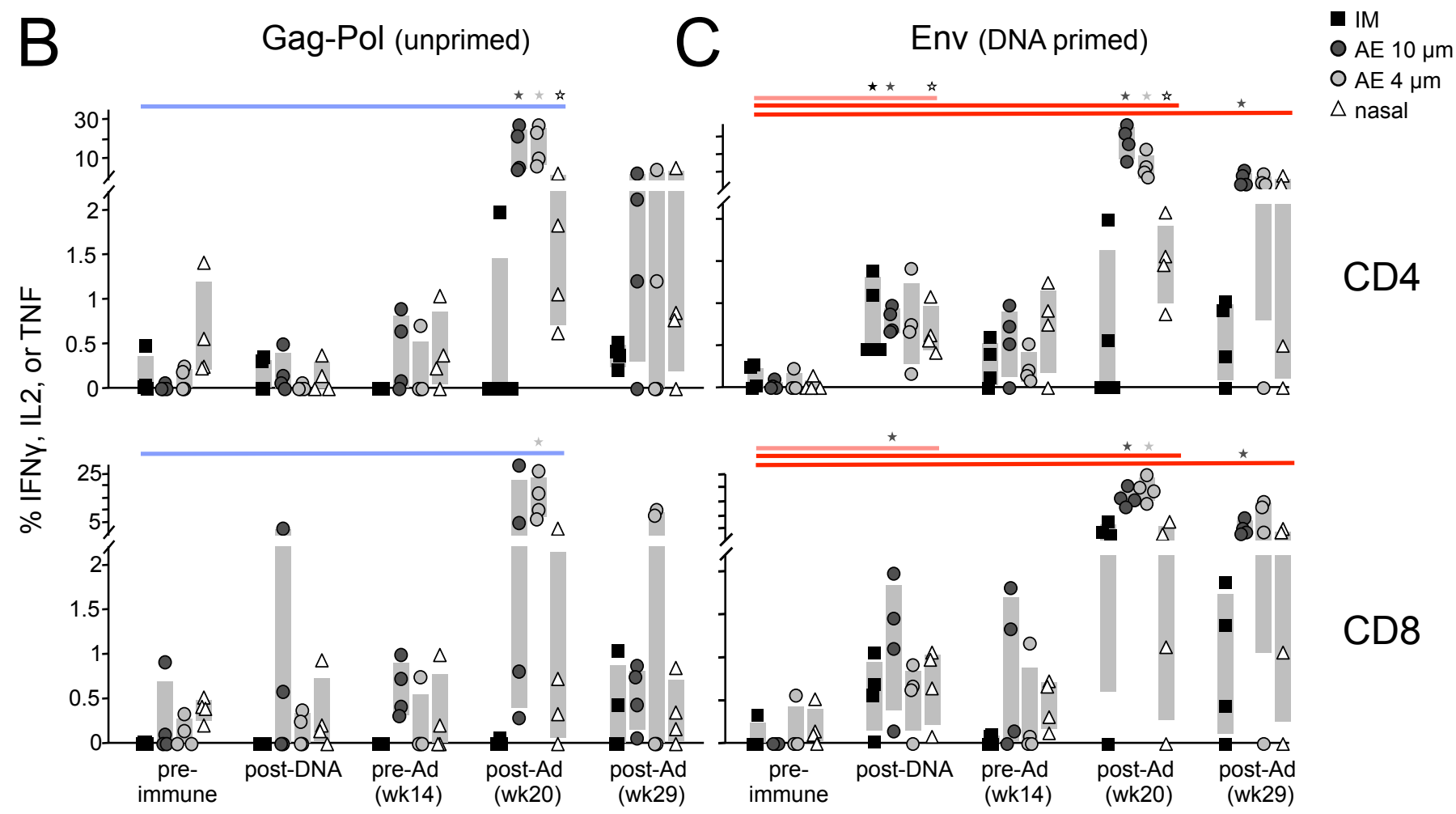

D
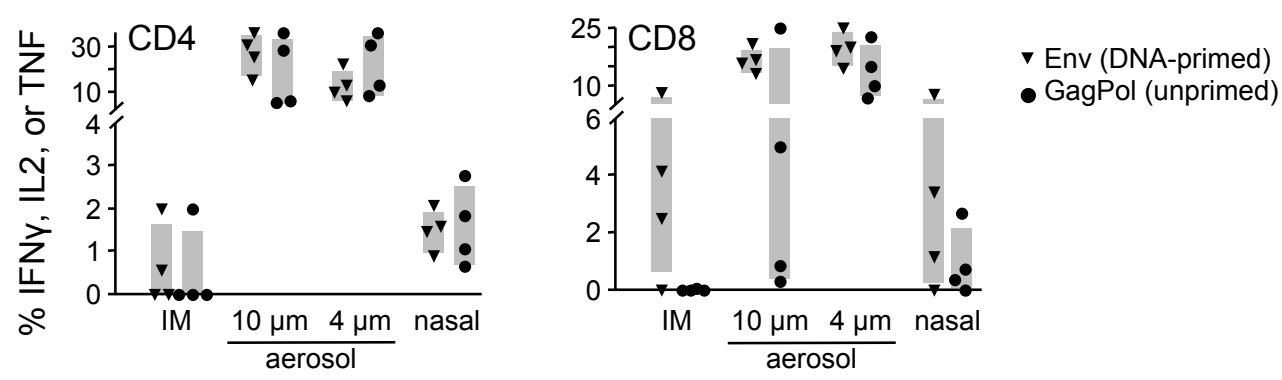
Figure 3
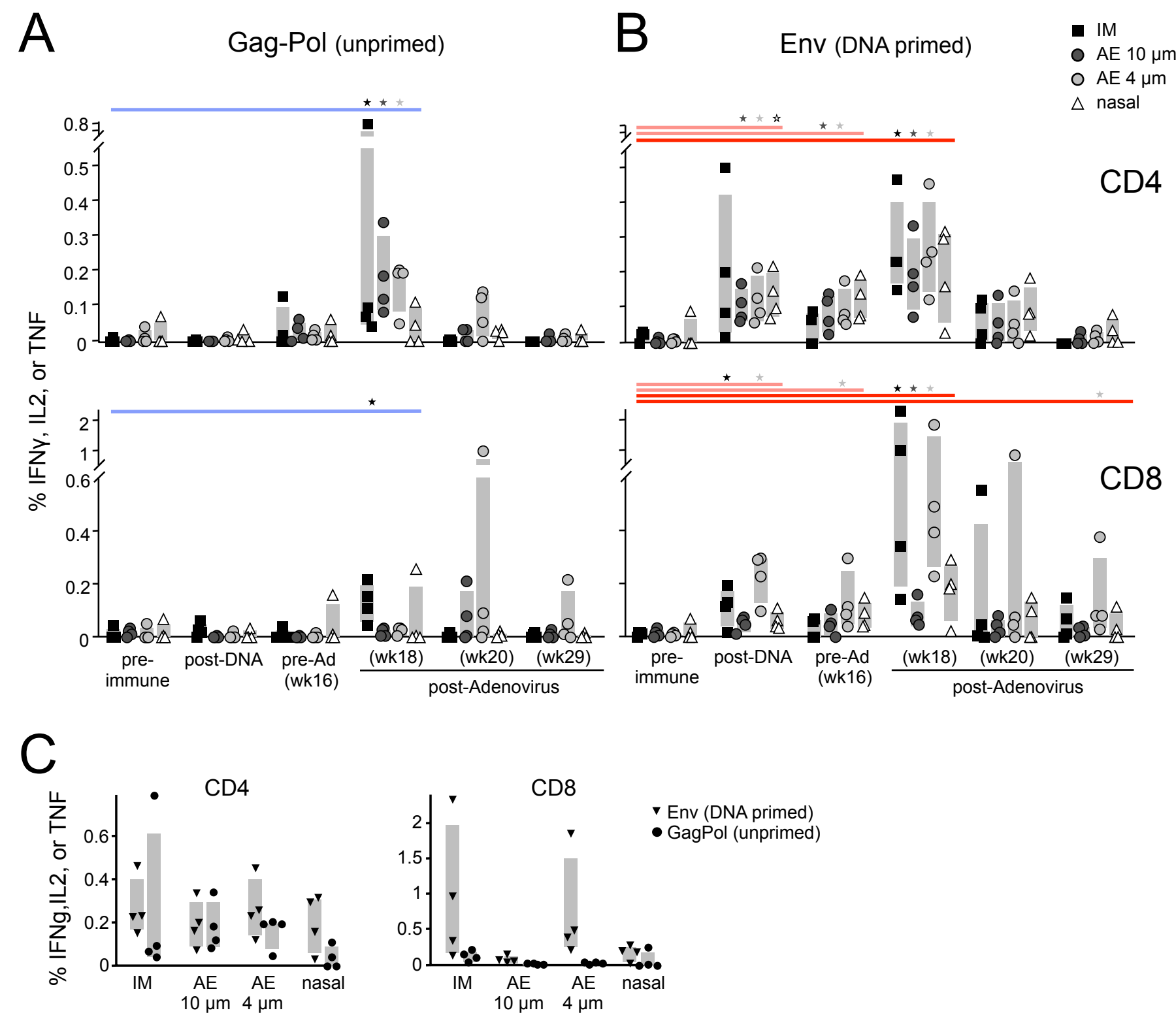


\section{Figure 4}
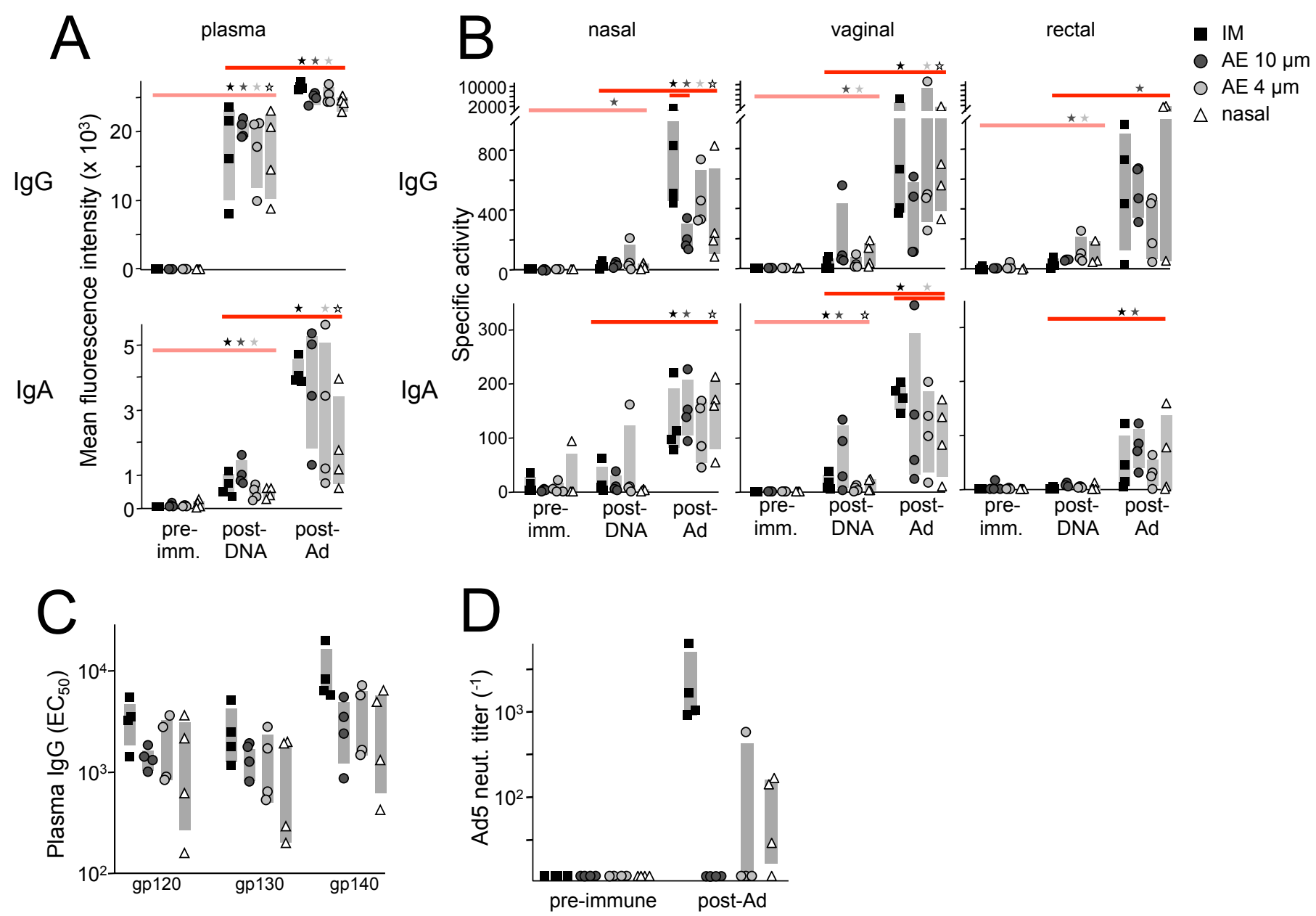